
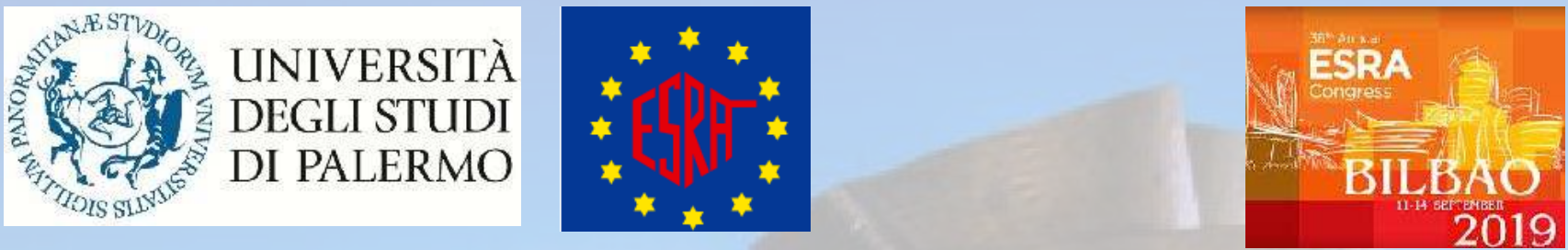

\title{
THE ROLE OF BILATERAL CONTINUOUS TRANSVERSUS ABDOMINIS PLANE BLOCK FOR POSTOPERATIVE ANALGESIA IN MAJOR ABDOMINAL SURGERY: A CASE SERIES
}

P. Giammanco1, A. Porto1, F. Quattrocchi1', M. Segreto1, S. Scifo1, F. Silvia1, D. Spina1, G. Torta1, M. Raineri1, D. Canzio2,

1Section of Anaesthesia- Analgesia- Emergency- Intensive Care. Palermo University Medical Center- Italy. A. Giarratano, Full Professor

2Department of Surgical- Oncological and Oral Science Di.Chir.On.S., Palermo- Via del Vespro 129.90127, Ital

\section{BACKGROUND AND AIM}

The use of loco-regional anesthesia aims at providing an adequate intra- and/or post-operative pain management for a broad spectrum of surgical interventions which historically have relayed on the use of opiod drugs.

On this fashion, the aim of our study is to prove that the use of bilateral continuous subcostal TAP block, in a multimodal opiod-sparing analgesic approach, provides a successful post-operative pain management for major abdominal surgical interventions. We adopted the NRS score as a reference and $\leq 6$ as an acceptable response.

\section{METHODS:}

We enrolled (16) patients undergoing major abdominal surgery. A primer infusion of $1 \mathrm{gr}$ paracetamol $+30 \mathrm{mg}$ ketoralac was administered 30 minutes prior to the end of the intervention. Moreover, in the immediate post-operative period, via US guidance, a $20 \mathrm{ml}$ bolus of $0.5 \%$ levobupivacaine was
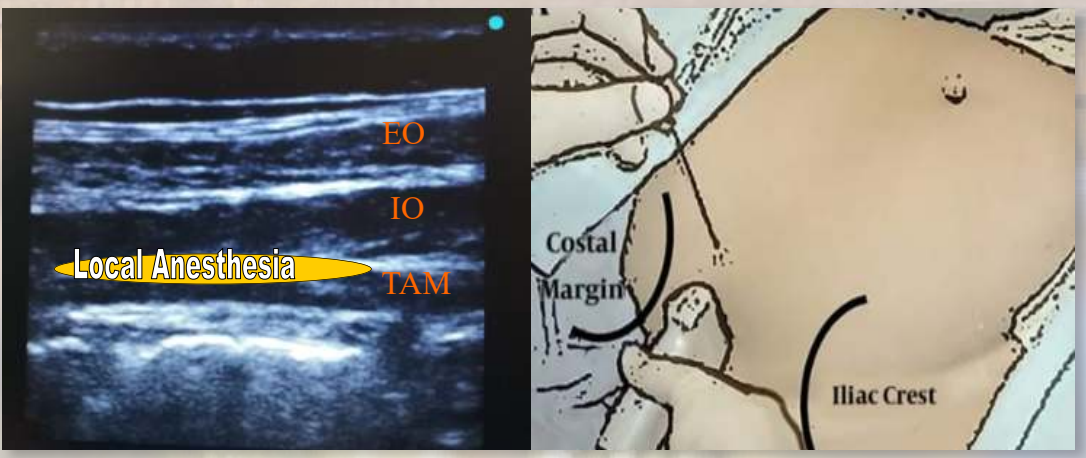
administered bilaterally. Then, a permanent catheter was placed on both sides with a $2 \mathrm{ml} / \mathrm{h}$ infusion of $0.5 \%$ levobupivacaine $(20 \mathrm{ml}$ in $28 \mathrm{ml}$ of $0.9 \% \mathrm{NaCl}$ ).

\section{RESULTS:}

In the 30-minutes post-operative period, the NRS score was $\leq 4$ for all of our patients. Then $\leq 3$ in the 6,12 and 24-hour post-operative period. None of our patients requested any supplementary analgesics.

\section{CONCLUSIONS:}

The major drawback of this study is the small size of our sample. Further studies might encourage the spread of this procedure as a new gold-standard for laparoscopic interventions (e.g. right hemicolectomy and laparocele) and its non-inferiority to epidural analgesia, the current gold-standard for laparotomic surgeries (especially left hemicolectomy).

\section{References:}

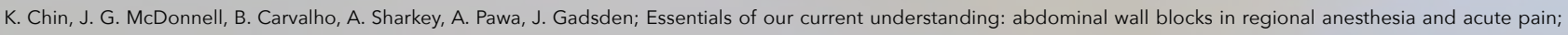
American Society of Regional Anesthesia and Pain Medicine; a. 2017

J. Ma, Y. Jiang, S. Tang, B. Wang, Q. Lian, Z. Xie, J. Li; Analgesic efficacy of ultrasound-guided subcostal transversus abdominis plane block; www.md-journal.com; a. 2017 